\title{
HESSIAN MANIFOLDS \\ OF NONPOSITIVE CONSTANT HESSIAN SECTIONAL CURVATURE
}

\author{
Hitoshi FuruhatA AND TAKASHI KuROSE
}

(Received December 5, 2011)

\begin{abstract}
We classify the maximal Hessian manifolds of constant Hessaian sectional curvature nonpositive.
\end{abstract}

1. Introduction. Hessian geometry is a close relative of Kählerian geometry, and connected with many interesting fields such as affine differential geometry and information geometry. It was founded and deeply studied by J. L. Koszul, H. Shima and others. A systematic introduction to Hessian geometry is given in the book [5]. In [4], Shima defined Hessian sectional curvatures and studied spaces of constant Hessian sectional curvature. In this paper, we give a characterization of certain important examples of such spaces as the standard models (Theorem 4.1 in the case of constant Hessian sectional curvature zero, and Theorem 5.2 in the case of constant Hessian sectional curvature negative).

Throughout this paper, let $M$ be an $n$-dimensional connected manifold, $\nabla$ an affine connection of torsion-free, and $g$ a Riemannian metric on $M$. All the objects are assumed to be smooth. We denote by $\Gamma(E)$ the set of sections of a vector bundle $E \rightarrow M$. For example, $\Gamma\left(T M^{(p, q)}\right)$ means the set of tensor fields of type $(p, q)$ on $M$. The torsion tensor field and the curvature tensor tensor field of $\nabla$ are denoted by $T^{\nabla} \in \Gamma\left(T M^{(1,2)}\right)$ and $R^{\nabla} \in \Gamma\left(T M^{(1,3)}\right)$, respectively.

DEFinition 1.1. A triplet $(M, \nabla, g)$ is called a Hessian manifold if (1) $\nabla g \in$ $\Gamma\left(T M^{(0,3)}\right)$ is symmetric, and (2) $R^{\nabla}=0$.

The name comes from the following facts. For an arbitrary point $p$ of a Hessian manifold $(M, \nabla, g)$, there exist a neighborhood $U$ of $p$ and a function $\varphi \in C^{\infty}(U)$ such that $\left.g\right|_{U}=\nabla d \varphi$, that is, the Riemannian metric is locally expressed as the Hessian matrix of a function with respect to an affine coordinate system. Besides, we should recall that a triplet $(M, \nabla, g)$ is called a statistical manifold if (1) in Definition 1.1 holds. This notion comes from information geometry (See [1] and [3]).

Definition 1.2. For a Hessian manifold $(M, \nabla, g)$, we denote the Levi-Civita connection of $g$ by $\nabla^{g}$ or $\widehat{\nabla}$. We use the latter when the metric is clearly specified. We then define

2000 Mathematics Subject Classification. Primary 53A15; Secondary 53C05, 53C25.

Key words and phrases. Hessian structure, constant curvature, multinomial distribution.

The first named author is partly supported by JSPS KAKENHI 21540059. The second named author is partly supported by JSPS KAKENHI 22540107. 
the difference tensor field $K:=K^{(\nabla, g)} \in \Gamma\left(T M^{(1,2)}\right)$ as

$$
K(X, Y):=\nabla_{X} Y-\widehat{\nabla}_{X} Y
$$

for all $X, Y \in \Gamma(T M)$.

Definition 1.3. Let $(M, \nabla, g)$ be a Hessian manifold and $c \in \boldsymbol{R} .(\nabla, g)$ is said to be of constant Hessian sectional curvature $c$ if

$$
\left(\nabla_{X} K\right)(Y, Z):=(\nabla K)(Y, Z ; X)=-\frac{c}{2}\{g(X, Y) Z+g(X, Z) Y\}
$$

for any $X, Y, Z \in \Gamma(T M)$. We abbreviate it as "of $\mathrm{CHC} c$ " in this paper.

We can construct a Kählerian metric $g^{T}$ on the tangent bundle of a Hessian manifold $M$ by the diagonal lifting (or the Sasaki lifting) and remark that $M$ is of CHC $c$ if and only if $\left(T M, g^{T}\right)$ is of constant holomorphic sectional curvature $-c$.

Proposition 1.4 ([4]). Let $(M, \nabla, g)$ be a Hessian manifold of CHC c. Then $(M, g)$ is a Riemannian manifold of constant curvature -c/4. In fact, the following holds:

$$
\begin{aligned}
R^{\widehat{\nabla}}(X, Y) Z & =-\frac{1}{2}\{(\nabla K)(Y, Z ; X)-(\nabla K)(Z, X ; Y)\} \\
& =-\frac{c}{4}\{g(Y, Z) X-g(X, Z) Y\} .
\end{aligned}
$$

We denote the Euclidean space, the sphere of curvature $\kappa>0$ (of radius $\kappa^{-1 / 2}$ with center $\left.0 \in \boldsymbol{R}^{n+1}\right)$, the hyperbolic space of curvature $\kappa<0$ by $\left(\boldsymbol{R}^{n}, g_{E}\right),\left(S^{n}(\kappa), g_{S}\right),\left(H^{n}(\kappa), g_{H}\right)$, respectively, and by $\left(\widetilde{M}^{n}(\kappa), \widetilde{g}\right)$ in general.

COROLlary 1.5. Let $(M, \nabla, g)$ be an n-dimensional simply-connected Hessian manifold of $C H C-4 \kappa$. Then there exists an isometric immersion ı of $M$ to $\tilde{M}^{n}(\kappa)$. In particular, $g$ is not complete if $\iota(M) \neq \widetilde{M}^{n}(\kappa)$.

\section{Examples of Hessian manifolds of CHC.}

EXAMPLE 2.1. The Euclidean space $\left(\boldsymbol{R}^{n}, \nabla^{g_{E}}, g_{E}\right)$ with the standard connection is a Hessian manifold of CHC 0.

EXAMPLE 2.2. The triplet $\left(\left(\boldsymbol{R}^{+}\right)^{n}, D, g\right)$ defined by

$$
\begin{aligned}
& \left(\boldsymbol{R}^{+}\right)^{n}:=\left\{y={ }^{t}\left(y^{1}, \ldots, y^{n}\right) \in \boldsymbol{R}^{n} ; y^{1}>0, \ldots, y^{n}>0\right\}, \\
& g:=\left.g_{E}\right|_{\left(\boldsymbol{R}^{+}\right)^{n}}=\sum_{j=1}^{n}\left(d y^{j}\right)^{2}, \\
& D: \quad D_{\partial / \partial y^{i}} \frac{\partial}{\partial y^{j}}=-\delta_{i j}\left(y^{j}\right)^{-1} \frac{\partial}{\partial y^{j}},
\end{aligned}
$$

is a Hessian manifold of CHC 0 . 
EXAMPLE 2.3. For $k \in\{0,1, \ldots, n\}$, the triplet $\left(N_{k}, \nabla_{k}, g_{k}\right)$ defined by

$$
\begin{aligned}
& N_{k}:=\left\{y={ }^{t}\left(y^{1}, \ldots, y^{n}\right) \in \boldsymbol{R}^{n} ; y^{n-k+1}>0, \ldots, y^{n}>0\right\}, \\
& g_{k}:=\left.g_{E}\right|_{N_{k}}=\sum_{j=1}^{n}\left(d y^{j}\right)^{2}, \\
& \nabla_{k}:=\nabla^{g_{k}}+K_{k}, \quad \text { where } K_{k}:=\sum_{j=n-k+1}^{n}\left(-y^{j}\right)^{-1} d y^{j} \otimes d y^{j} \otimes \frac{\partial}{\partial y^{j}},
\end{aligned}
$$

is also a Hessian manifold of CHC 0.

We remark that $\left(N_{k}, \nabla_{k}, g_{k}\right)$ coincides with $\left(\boldsymbol{R}^{n}, \nabla^{g_{E}}, g_{E}\right)$ when $k=0$, and $\left(\left(\boldsymbol{R}^{+}\right)^{n}\right.$, $D, g)$ when $k=n$.

Let $(\tilde{M}, \widetilde{\nabla}, \tilde{g})$ be a Hessian manifold, and $f: M \rightarrow \widetilde{M}$ an immersion. We define $g$ and $\nabla$ on $M$ by

$$
g=f^{*} \widetilde{g}, \quad g\left(\nabla_{X} Y, Z\right)=\widetilde{g}\left(\widetilde{\nabla}_{X} f_{*} Y, f_{*} Z\right)
$$

for any $X, Y, Z \in \Gamma(T M)$, where the connection induced from $\widetilde{\nabla}$ by $f$ on the induced bundle $f^{*} T \widetilde{M} \rightarrow M$ is denoted by the same symbol $\widetilde{\nabla}$. Then the pair $(\nabla, g)$ is a statistical structure on $M$, which is called the one induced by $f$ from $(\widetilde{\nabla}, \widetilde{g})$ (See [2]).

EXAMPLE 2.4. Let $f: S_{+}^{n}(\kappa):=S^{n}(\kappa) \cap\left(\boldsymbol{R}^{+}\right)^{n+1} \rightarrow\left(\boldsymbol{R}^{+}\right)^{n+1}$ be the inclusion, and $\left(\nabla^{S_{+}}, g_{S_{+}}\right)$the statistical structure induced by $f$ from $(D, g)$ in Example 2.2. Then the triplet $\left(S_{+}^{n}(\kappa), \nabla^{S_{+}}, g_{S_{+}}\right)$is a Hessian manifold of CHC $-4 \kappa(<0)$.

We set

$$
\tilde{f}: \boldsymbol{R}^{n} \ni x={ }^{t}\left(x^{1}, \ldots, x^{n}\right) \mapsto \kappa^{-1 / 2} \Lambda^{-1 / 2}(x)^{t}\left(e^{x^{1} / 2}, \ldots, e^{x^{n} / 2}, 1\right) \in\left(\boldsymbol{R}^{+}\right)^{n+1},
$$

where $\Lambda(x):=1+\sum_{j=1}^{n} e^{x^{j}}$. Then it is easy to show that $\widetilde{f}\left(\boldsymbol{R}^{n}\right)=S_{+}^{n}(\kappa)$, and $\left(x^{1}, \ldots, x^{n}\right)$ are affine coordinates of $S_{+}^{n}(\kappa)$ with respect to $\nabla^{S_{+}}$, that is, $\nabla^{S_{+}}\left(\partial / \partial x^{j}\right)=0$. The metric is given in these coordinates by

$$
\widetilde{f}^{*} g_{E}=g_{S_{+}}=(4 \kappa)^{-1} \Lambda^{-1}(x) \sum\left\{\delta_{i j} e^{x^{i}}-\Lambda^{-1}(x) e^{x^{i}} e^{x^{j}}\right\} d x^{i} \otimes d x^{j} .
$$

EXAMPLE 2.5. For $x \in\{1,2, \ldots, n+1\}$ and $\Delta:=\left\{\eta={ }^{t}\left(\eta^{1}, \ldots, \eta^{n}\right) \in \boldsymbol{R}^{n} ; \eta^{j}>\right.$ $\left.0, \sum_{j=1}^{n} \eta^{j}<1\right\}$, the probability density function of the $(n+1)$-nomial distribution is given by

$$
\begin{aligned}
p(x ; \eta) & := \begin{cases}\eta^{i} & x=i \in\{1,2, \ldots, n\}, \\
1-\sum_{j=1}^{n} \eta^{j} & x=n+1\end{cases} \\
& =\exp \left(\sum_{i=1}^{n} \log \frac{\eta^{i}}{1-\sum_{j=1}^{n} \eta^{j}} \delta_{i x}-\log \frac{1}{1-\sum_{j=1}^{n} \eta^{j}}\right) .
\end{aligned}
$$

We denote the Fisher information metric on $\Delta$ by $g_{F}$, and the exponential connection on $\Delta$ by $\nabla^{(e)}\left(=\nabla^{(1)}\right)$ (See [1]). Then $\left(\Delta, \nabla^{(e)}, g_{F}\right)$ is a Hessian manifold of CHC -1 . 
It is known by [4] that there exists a diffeomorphism $\Phi: \Delta \rightarrow S_{+}^{n}(1 / 4)$ such that

$$
\Phi^{*} \nabla^{S_{+}}=\nabla^{(e)} \quad \text { and } \quad \Phi^{*} g_{S_{+}}=g_{F} .
$$

\section{Preliminaries.}

Proposition 3.1. Let $(M, g)$ be a Riemannian manifold of constant curvature $\kappa$. For $K \in \Gamma\left(T M^{(1,2)}\right)$, we set $\nabla:=\widehat{\nabla}+K$. The triplet $(M, \nabla, g)$ is a Hessian manifold of $C H C$ $-4 \kappa$ if and only if $K$ satisfies the following four conditions:

$$
\begin{gathered}
K(X, Y)=K(Y, X), \\
g(K(X, Y), Z)=g(Y, K(X, Z)), \\
\kappa\{g(Y, Z) X-g(X, Z) Y\}=K(Y, K(X, Z))-K(X, K(Y, Z)), \\
(\widehat{\nabla} K)(X, Y ; Z)=2 \kappa\{g(Z, X) Y+g(Z, Y) X\} \\
-K(Z, K(X, Y))+K(K(Z, X), Y)+K(X, K(Z, Y)) .
\end{gathered}
$$

Proof. We use the following formulas (3.5) through (3.9). Equation (3.6) holds under (3.1). Equation (3.9) holds under (3.1) and (3.4).

$$
\begin{gathered}
K(X, Y)-K(Y, X)=T^{\nabla}(X, Y), \\
g(X, K(Y, Z))-g(K(Y, X), Z) \\
\quad=(\nabla g)(Y, Z ; X)-(\nabla g)(X, Z ; Y),
\end{gathered}
$$

$(\widehat{\nabla} K)(X, Y ; Z)$

$$
\begin{aligned}
+ & K(Z, K(X, Y))-K(K(Z, X), Y)-K(X, K(Z, Y)) \\
= & (\nabla K)(X, Y ; Z), \\
R^{\nabla}(X, Y) Z & \\
= & R^{\widehat{\nabla}}(X, Y) Z+(\widehat{\nabla} K)(Y, Z ; X)-(\widehat{\nabla} K)(X, Z ; Y) \\
& -K(Y, K(X, Z))+K(X, K(Y, Z)), \\
= & -\kappa\{g(Y, Z) X-g(X, Z) Y\} \\
& +K(Y, K(X, Z))-K(X, K(Y, Z)) .
\end{aligned}
$$

Assuming (3.1) through (3.4), we derive that $(M, \nabla, g)$ is a Hessian manifold of CHC $-4 \kappa$. By (3.5), (3.1) implies that $\nabla$ is of torsion free. By (3.6), (3.2) implies that $\nabla g$ is symmetric. By (3.7), (3.4) implies that $(\nabla, g)$ is of $\mathrm{CHC}-4 \kappa$. By (3.9), (3.3) implies that $\nabla$ is flat. The converse is proved in the similar way.

The formulas above are derived by direct calculations. Here we give the proof of only (3.8) and (3.9) as follows. Since $\nabla_{Y} Z=\widehat{\nabla}_{Y} Z+K(Y, Z)$, we have

$$
\nabla_{X} \nabla_{Y} Z=\widehat{\nabla}_{X} \widehat{\nabla}_{Y} Z+\widehat{\nabla}_{X} K(Y, Z)+K\left(X, \widehat{\nabla}_{Y} Z\right)+K(X, K(Y, Z)),
$$


and

$$
-\nabla_{[X, Y]} Z=-\widehat{\nabla}_{[X, Y]} Z-K\left(\widehat{\nabla}_{X} Y-\widehat{\nabla}_{Y} X, Z\right) .
$$

Then

$$
\begin{aligned}
R^{\nabla}( & X, Y) Z \\
= & \nabla_{X} \nabla_{Y} Z-\nabla_{Y} \nabla_{X} Z-\nabla_{[X, Y]} Z \\
= & R^{\widehat{\nabla}}(X, Y) Z \\
& +\widehat{\nabla}_{X} K(Y, Z)+K\left(X, \widehat{\nabla}_{Y} Z\right)+K(X, K(Y, Z)) \\
& -\widehat{\nabla}_{Y} K(X, Z)-K\left(Y, \widehat{\nabla}_{X} Z\right)-K(Y, K(X, Z)) \\
& -K\left(\widehat{\nabla}_{X} Y, Z\right)+K\left(\widehat{\nabla}_{Y} X, Z\right),
\end{aligned}
$$

which is the right-hand side of (3.8). Since $g$ is of constant curvature $\kappa$, we have, using (3.4) and (3.1),

$$
\begin{aligned}
\text { RHS of } & (3.8) \\
= & \kappa\{g(Y, Z) X-g(X, Z) Y\} \\
+ & {[2 \kappa\{g(X, Y) Z+g(X, Z) Y\}} \\
& -K(X, K(Y, Z))+K(K(X, Y), Z)+K(Y, K(X, Z))] \\
- & {[2 \kappa\{g(Y, X) Z+g(Y, Z) X\}} \\
& -K(Y, K(X, Z))+K(K(Y, X), Z)+K(X, K(Y, Z))] \\
- & K(Y, K(X, Z))+K(X, K(Y, Z)),
\end{aligned}
$$

which is the right-hand side of (3.9).

Proposition 3.2. Let $(M, g)$ be a Riemannian manifold of constant curvature $\kappa$. Suppose that $K \in \Gamma\left(T M^{(1,2)}\right)$ satisfies the differential equation (3.4) on $M$. If the algebraic conditions (3.1) through (3.3) hold at some point of $M$, then they hold on $M$, that is, the triplet $(M, \nabla:=\widehat{\nabla}+K, g)$ is a Hessian manifold of $C H C-4 \kappa$.

Proof. As the first step, we define $\alpha \in \Gamma\left(T M^{(1,2)}\right)$ by

$$
\alpha(X, Y):=K(X, Y)-K(Y, X),
$$

and get, by using (3.4),

$$
\begin{aligned}
& (\widehat{\nabla} \alpha)(X, Y ; Z) \\
& \quad=-K(Z, \alpha(X, Y))+\alpha(X, K(Z, Y))-\alpha(Y, K(Z, X))
\end{aligned}
$$

for all $X, Y, Z \in \Gamma(T M)$. If $\alpha$ vanishes at some point, $\alpha=0$ is the unique solution of the differential equation (3.10) with the condition. It implies that (3.1) holds on $M$. The formula (3.10) is obtained by a direct calculation.

As the second step, we define $\beta \in \Gamma\left(T M^{(0,3)}\right)$ and $\gamma \in \Gamma\left(T M^{(1,3)}\right)$ by

$$
\begin{aligned}
& \beta(X, Y, Z):=g(K(X, Y), Z)-g(Y, K(X, Z)), \\
& \gamma(X, Y, Z):=\kappa g(Y, Z) X+K(X, K(Y, Z))
\end{aligned}
$$




$$
-\{\kappa g(X, Z) Y+K(Y, K(X, Z))\}
$$

and show that $\beta$ and $\gamma$ vanish identically in the same way. We can calculate the following differential equations by the conditions (3.1) and (3.4):

$$
\begin{aligned}
&(\widehat{\nabla} \beta)(X, Y, Z ; V) \\
&= 2 \beta(Y, K(Z, V), X)-2 \beta(Z, K(Y, V), X) \\
&-\beta(V, K(X, Y), Z)+\beta(X, K(V, Y), Z) \\
&+ \beta(V, K(X, Z), Y)-\beta(X, K(V, Z), Y) \\
&+\beta(Y, K(V, X), Z)-\beta(Z, K(V, X), Y) \\
&-2 g(\gamma(Y, Z, V), X), \\
&(\widehat{\nabla} \gamma)(X, Y, Z ; V) \\
&=K(V,-\gamma(X, Y, Z))+\gamma(X, Y, K(Z, V)) \\
&+\gamma(X, K(Y, V), Z)+\gamma(K(X, V), Y, Z) \\
&+\gamma\left(\widehat{\nabla}_{V} X, Y, Z\right)+\kappa\{\beta(Z, Y, V)-\beta(Y, V, Z)\} X \\
&-\kappa\{\beta(Z, X, V)-\beta(X, V, Z)\} Y .
\end{aligned}
$$

The same argument to the first step works.

Here we give the proof of (3.11). The formula (3.12) is obtained in the similar calculation. By definition, we have

$$
\begin{aligned}
- & g(\gamma(Y, Z, V), X) \\
= & \kappa\{g(Y, V) g(Z, X)-g(Z, V) g(Y, X)\} \\
& +g(K(Z, K(Y, V)), X)-g(K(Y, K(Z, V)), X) \\
= & \kappa\{g(Y, V) g(Z, X)-g(Z, V) g(Y, X)\} \\
& +g(K(Y, V), K(Z, X))-g(K(Z, V), K(Y, X)) \\
& +\beta(Z, K(Y, V), X)-\beta(Y, K(Z, V), X) .
\end{aligned}
$$

We calculate by using (3.4) and (3.1),

$$
\begin{aligned}
(\widehat{\nabla} \beta) & (X, Y, Z ; V) \\
= & g((\widehat{\nabla} K)(X, Y ; V), Z)-g(Y,(\widehat{\nabla} K)(X, Z ; V)) \\
= & 2 \kappa\{g(V, Y) g(X, Z)-g(V, Z) g(X, Y)\} \\
& -g(K(V, K(X, Y)), Z)+g(K(X, K(V, Y)), Z) \\
& +g(K(V, K(X, Z)), Y)-g(K(X, K(V, Z)), Y) \\
& +g(K(Y, K(V, X)), Z)-g(K(Z, K(V, X)), Y),
\end{aligned}
$$


and from (3.13) that

$$
\begin{aligned}
=2 & -g(K(Y, V), K(Z, X))+g(K(Z, V), K(Y, X)) \\
& -\beta(Z, K(Y, V), X)+\beta(Y, K(Z, V), X) \\
& -g(\gamma(Y, Z, V), X)\} \\
- & g(K(X, Y), K(V, Z))-\beta(V, K(X, Y), Z) \\
+ & g(K(V, Y), K(X, Z))+\beta(X, K(V, Y), Z) \\
+ & g(K(X, Z), K(V, Y))+\beta(V, K(X, Z), Y) \\
- & g(K(V, Z), K(X, Y))-\beta(X, K(V, Z), Y) \\
+ & g(K(V, X), K(Y, Z))+\beta(Y, K(V, X), Z) \\
& -g(K(V, X), K(Z, Y))-\beta(Z, K(V, X), Y) \\
= & \mathrm{RHS} \text { of }(3.11) .
\end{aligned}
$$

LEMma 3.3. The differential equation (3.4) for $K$ satisfies the integrability condition provided (3.1) through (3.3).

PROOF. We have only to derive the following Ricci's identity from Equation (3.4) :

$$
\begin{aligned}
\left(\widehat{\nabla}^{2} K\right)(X, Y ; V, W)-\left(\widehat{\nabla}^{2} K\right)(X, Y ; W, V) \\
=R^{\widehat{\nabla}}(W, V) K(X, Y) \\
\quad-K\left(R^{\widehat{\nabla}}(W, V) X, Y\right)-K\left(X, R^{\widehat{\nabla}}(W, V) Y\right) .
\end{aligned}
$$

The left hand side of (3.14) is calculated as follows:

$$
\begin{aligned}
&\left(\widehat{\nabla}^{2} K\right)(X, Y ; V, W)-\left(\widehat{\nabla}^{2} K\right)(X, Y ; W, V) \\
&=\kappa g(W, Y) K(V, X)+g(K(V, X), Y) W \\
&+g(W, X) K(V, Y)\} \\
&- \kappa\{g(V, Y) K(W, X)+g(K(W, X), Y) V \\
&+g(V, X) K(W, Y)\} .
\end{aligned}
$$

In fact, by (3.3) we can get

$$
\begin{aligned}
\left(\widehat{\nabla}^{2} K\right) & (X, Y ; V, W) \\
= & (\widehat{\nabla} K)(K(V, X), Y ; W)+K((\widehat{\nabla} K)(V, X ; W), Y) \\
= & \kappa\{g(K(V, X), Y) W+g(W, Y) K(V, X) \\
& +2 g(K(V, X), W) Y\}+K(K(W, K(V, X)), Y) \\
& +K(\kappa\{g(V, X) W+g(W, X) V+2 g(W, V) X\} \\
& \quad+K(K(W, V), X), Y) \\
= & \kappa\{g(W, Y) K(V, X)+g(K(V, X), Y) W\} \\
+ & K(K(W, K(V, X)), Y)+S(X, Y, V, W),
\end{aligned}
$$


where $S \in \Gamma\left(T M^{(1,4)}\right)$ is a term symmetric with respect to $V$ and $W$, which is given by

$$
\begin{aligned}
& S(X, Y, V, W) \\
&=\kappa\{2 g(K(V, X), W) Y+2 g(V, W) K(X, Y) \\
&+ \\
&+g(W, X) K(V, Y)+g(V, X) K(W, Y)\} \\
&+ K(K(K(W, V), X), Y) .
\end{aligned}
$$

It is easy to calculate (3.15) from (3.16) and (3.3). On the other hand, the right side of (3.14) is calculated as follows:

$$
\begin{aligned}
R^{\widehat{\nabla}}(W, V) K(X, Y)-K\left(R^{\widehat{\nabla}}(W, V) X, Y\right)-K\left(X, R^{\widehat{\nabla}}(W, V) Y\right) \\
=\kappa\{g(V, K(X, Y)) W-g(W, K(X, Y)) V\} \\
\quad-\kappa\{g(V, X) K(W, Y)-g(W, X) K(V, Y)\} \\
\quad-\kappa\{g(V, Y) K(X, W)-h(W, Y) K(X, V)\},
\end{aligned}
$$

which coincides with (3.15), and thus, we obtain the equation (3.14).

The uniqueness derived from this integrability condition implies

COROLlary 3.4. Let $(M, \nabla, g),(\tilde{M}, \widetilde{\nabla}, \tilde{g})$ be n-dimensional simply-connected Hessian manifolds of $C H C-4 \kappa$ with difference tensor fields $K, \widetilde{K}$, respectively. Let $\iota: M \rightarrow \widetilde{M}$ be an isometric immersion. If $\iota^{*} \widetilde{K}=K$ at some point, then $\iota^{*} \widetilde{\nabla}=\nabla$ and $\iota^{*} \widetilde{g}=g$ on $M$.

4. Hessian manifolds of CHC zero. In this section, we show that the $n$-dimensional Hessian manifolds $\left(N_{k}, \nabla_{k}, g_{k}\right)$ in Example 2.3 are the only maximal ones of CHC zero. Namely, we prove the following theorem.

THEOREM 4.1. Let $(M, \nabla, g)$ be an $n$-dimensional simply-connected Hessian manifold of CHC 0 . Then there exist $k \in\{0,1, \ldots, n\}$ and an immersion $\iota: M \rightarrow N_{k}$ such that $\iota^{*} \nabla_{k}=\nabla$ and $\iota^{*} g_{k}=g$.

Proof. Take a point $p \in M$ and fix it. We claim that there exist orthonormal basis $\left\{e_{1}, \ldots, e_{n}\right\}$ of $T_{p} M$ with respect to $g$, and real numbers $\lambda_{j}$ such that $0 \geq \lambda_{1} \geq \cdots \geq \lambda_{n}$ and $K\left(e_{i}, e_{j}\right)=\lambda_{i} \delta_{i j} e_{j}$.

By (3.2), a linear transformation $K(X, \cdot): T_{p} M \rightarrow T_{p} M$ is symmetric with respect to $g$ for any $X \in T_{p} M$. Since linear transformations $K(X, \cdot)$ and $K(Y, \cdot)$ are mutually commutable for any $X, Y \in T_{p} M$ by (3.3), they are simultaneously diagonalizable. Accordingly, there exist orthonormal basis $\left\{\widetilde{e}_{1}, \ldots, \widetilde{e}_{n}\right\}$ of $T_{p} M$ and $\tilde{\lambda}_{i j} \in \boldsymbol{R}$ such that $K\left(\widetilde{e}_{i}, \widetilde{e}_{j}\right)=\widetilde{\lambda}_{i j} \widetilde{e}_{j}$. By (3.1), $\tilde{\lambda}_{i j}$ is written as $\tilde{\lambda}_{i j}=\tilde{\lambda}_{i} \delta_{i j}$ for some $\tilde{\lambda}_{i} \in \boldsymbol{R}$. In the case that $\tilde{\lambda}_{i}>0$, we set $e_{i}:=-\widetilde{e}_{i}$ and $\lambda_{i}:=-\tilde{\lambda}_{i}(<0)$. Otherwise, we set $e_{i}:=\widetilde{e}_{i}$ and $\lambda_{i}:=\tilde{\lambda}_{i}(\leq 0)$. Renumbering them if necessary, we may assume that

$$
0 \geq \lambda_{1} \geq \cdots \geq \lambda_{n} \text { and } K\left(e_{i}, e_{j}\right)=\lambda_{i} \delta_{i j} e_{j}
$$


Let $k \in\{0,1, \ldots, n\}$ be the minimal number such that $0=\lambda_{1}=\cdots=\lambda_{n-k}$. From Corollary 1.5, we have an isometric immersion $\iota:(M, g) \rightarrow\left(\boldsymbol{R}^{n}, g_{E}\right)$, and may assume that

$$
\begin{aligned}
& \iota(p)=\left[\begin{array}{c}
0_{n-k} \\
\left(-\lambda_{n-k+1}\right)^{-1} \\
\vdots \\
\left(-\lambda_{n}\right)^{-1}
\end{array}\right] \in N_{k} \subset \boldsymbol{R}^{n} \\
& \text { and }(d \iota)_{p}\left(e_{i}\right)=\left(\frac{\partial}{\partial y^{i}}\right)_{\iota(p)}, \quad i=1, \ldots, n .
\end{aligned}
$$

We next claim that $(d \iota)_{p} K\left(e_{i}, e_{j}\right)=K_{k}\left((d \iota)_{p} e_{i},(d \iota)_{p} e_{j}\right)$. In fact, it is easy to see that both sides vanish when $i \neq j$ or $i=j \leq n-k$. By definition, we have that each side is $\lambda_{i}\left(\partial / \partial y^{i}\right)_{\iota(p)}$ when $i=j>n-k$.

Using Corollary 3.4, we complete the proof of the theorem.

5. Hessian manifolds of CHC negative. In this section, we show that $\left(S_{+}^{n}(\kappa), \nabla^{S_{+}}\right.$, $\left.g_{S_{+}}\right)$in Example 2.4 is the only maximal Hessian manifolds of $\mathrm{CHC}-4 \kappa<0$. Accordingly, the Hessian manifold consisting of multinomial distributions is characterized as the maximal one of $\mathrm{CHC}-1$.

Proposition 5.1. Let $(M, \nabla, g)$ be an n-dimensional Hessian manifold of $C H C$ $-4 \kappa<0$. We define the affine connection $\widetilde{\nabla}$ on the Riemannian cone $\left(\tilde{M}:=M \times \boldsymbol{R}^{+}, \widetilde{g}:=\right.$ $\left.t^{2} \kappa g+d t^{2}\right)$ over $(M, g)$ by

$$
\begin{aligned}
\widetilde{\nabla}_{X} Y & =\nabla_{X} Y-2 t \kappa g(X, Y) T, \\
\widetilde{\nabla}_{Z} T & =\widetilde{\nabla}_{T} Z=0, \\
\widetilde{\nabla}_{T} T & =-t^{-1} T,
\end{aligned}
$$

where $T=d / d t$ and we identify vector fields $X, Y, Z$ on $M$ with those on $\tilde{M}$ in the natural way. Then $(\widetilde{M}, \widetilde{\nabla}, \widetilde{g})$ is an $(n+1)$-dimensional Hessian manifold of $C H C 0$.

PROOF. The following formulas (5.1) through (5.6) hold for vector fields $T, X, Y, Z$ on $\widetilde{M}$ as above:

$$
\begin{aligned}
(\widetilde{\nabla} \widetilde{g})(Y, Z ; X) & =t^{2} \kappa(\nabla g)(Y, Z ; X), \\
(\widetilde{\nabla} \widetilde{g})(X, Y ; T) & =(\widetilde{\nabla} \widetilde{g})(X, T ; Y)=(\widetilde{\nabla} \widetilde{g})(T, X ; Y) \\
& =2 t \kappa g(X, Y), \\
(\widetilde{\nabla} \widetilde{g})(X, T ; T) & =(\widetilde{\nabla} \widetilde{g})(T, X ; T)=(\widetilde{\nabla} \widetilde{g})(T, T ; X)=0, \\
(\widetilde{\nabla} \widetilde{g})(T, T ; T) & =2 t^{-1}, \\
& T^{\widetilde{\nabla}}(X, Y)=T^{\nabla}(X, Y), \\
& T^{\widetilde{\nabla}}(T, \cdot)=0,
\end{aligned}
$$




$$
\begin{aligned}
& R^{\widetilde{\nabla}}(X, Y) Z=R^{\nabla}(X, Y) Z, \\
& R^{\widetilde{\nabla}}(\cdot, \cdot) T=R^{\widetilde{\nabla}}(T, \cdot) \cdot=0, \\
& \nabla_{X}^{\widetilde{g}} Y=\nabla_{X}^{g} Y-t \kappa g(X, Y) T, \\
& \nabla_{X}^{\widetilde{g}} T=\nabla_{T}^{\widetilde{g}} X=t^{-1} X, \\
& \nabla_{T}^{\widetilde{g}} T=0,
\end{aligned}
$$

$$
\begin{aligned}
& \widetilde{K}(X, Y)=K(X, Y)-t \kappa g(X, Y) T, \\
& \widetilde{K}(X, T)=-t^{-1} X, \quad \widetilde{K}(T, T)=-t^{-1} T,
\end{aligned}
$$

$$
\begin{aligned}
& (\widetilde{\nabla} \widetilde{K})(Y, Z ; X) \\
& \quad=(\nabla K)(Y, Z ; X)-2 \kappa\{g(X, Y) Z+g(X, Z) Y\}, \\
& (\widetilde{\nabla} \widetilde{K})(T, \cdot ; \cdot)=(\widetilde{\nabla} \widetilde{K})(\cdot, T ; \cdot)=(\widetilde{\nabla} \widetilde{K})(\cdot, \cdot ; T)=0 .
\end{aligned}
$$

Equations (5.1), (5.2) and (5.3) show that $(\tilde{M}, \widetilde{\nabla}, \widetilde{g})$ is a Hessian manifold. Equations (5.6) shows that it is of $\mathrm{CHC} 0$.

THEOREM 5.2. Let $(M, \nabla, g)$ be an n-dimensional simply-connected Hessian manifold of $C H C-4 \kappa<0$. Then there exists an immersion $\iota: M \rightarrow S_{+}^{n}(\kappa)$ such that $\iota^{*} \nabla^{S_{+}}=\nabla$ and $\iota^{*} g_{S_{+}}=g$.

Proof. Let $(\tilde{M}, \widetilde{\nabla}, \tilde{g})$ be the $(n+1)$-dimensional Hessian manifold as in Proposition 5.1. If we set $\eta(x):=\left(x, \kappa^{-1 / 2}\right)$ for $x \in M$, then $(\nabla, g)$ is induced by $\eta: M \rightarrow \widetilde{M}$ from $(\widetilde{\nabla}, \tilde{g})$. Let $\left(\left(\boldsymbol{R}^{+}\right)^{n+1}=N_{n+1}, D=\nabla_{n+1}, g_{n+1}=\left.\langle\cdot, \cdot\rangle\right|_{\left(\boldsymbol{R}^{+}\right)^{n+1}}\right)$ be the $(n+1)$-dimensional Hessian manifold as in Examples 2.2 and $2.3(k=n+1)$, where $\langle\cdot, \cdot\rangle:=g_{E}$ is the Euclidean metric. We denote by $f$ the natural inclusion of the hypersphere $S^{n}(\kappa)$ of radius $\kappa^{-1 / 2}$ with center 0 into $\boldsymbol{R}^{n+1}$ and also its restriction on $S_{+}^{n}(\kappa)$. We recall that $\left(\nabla^{S_{+}}, g_{S_{+}}\right)$is induced by $f: S_{+}^{n}(\kappa) \rightarrow\left(\boldsymbol{R}^{+}\right)^{n+1}$ from $\left(D, g_{n+1}\right)$. To prove the theorem, we construct immersions $\varphi: \widetilde{M} \rightarrow\left(\boldsymbol{R}^{+}\right)^{n+1}$ and $\iota: M \rightarrow S_{+}^{n}(\kappa)$ such that $\varphi^{*} D=\widetilde{\nabla}, \varphi^{*} g_{n+1}=\widetilde{g}$ and the following diagram is commutative.

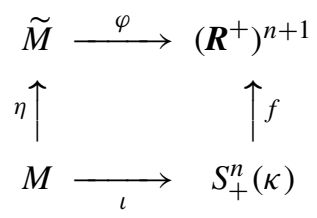

By Proposition 5.1, $(\tilde{M}, \widetilde{\nabla}, \widetilde{g})$ is of CHC 0. By Theorem 4.1, there exist $k \in\{0,1, \ldots$, $n+1\}$ and $\varphi: \widetilde{M} \rightarrow N_{k} \subset \boldsymbol{R}^{n+1}$ such that $\varphi^{*} \nabla_{k}=\widetilde{\nabla}$ and $\varphi^{*} g_{k}=\widetilde{g}$.

As the first step, we claim that $\varphi$ is given in the form $\varphi(x, t)=t \sqrt{\kappa} f \circ \iota(x)+c$ for some $\iota: M \rightarrow S^{n}(\kappa)$ and $c \in \boldsymbol{R}^{n+1}$. 
From the definition of $\tilde{g}$, we have that

$$
1=\left\langle\frac{\partial \varphi}{\partial t}, \frac{\partial \varphi}{\partial t}\right\rangle, \quad 0=\left\langle\frac{\partial \varphi}{\partial t}, \frac{\partial \varphi}{\partial x^{i}}\right\rangle, \quad t^{2} \kappa g_{i j}=\left\langle\frac{\partial \varphi}{\partial x^{i}}, \frac{\partial \varphi}{\partial x^{j}}\right\rangle
$$

and, by differentiating them, that

$$
0=\left\langle\frac{\partial^{2} \varphi}{\partial t \partial t}, \frac{\partial \varphi}{\partial t}\right\rangle, \quad 0=\left\langle\frac{\partial^{2} \varphi}{\partial x^{i} \partial t}, \frac{\partial \varphi}{\partial t}\right\rangle, \quad 0=\left\langle\frac{\partial^{2} \varphi}{\partial t \partial t}, \frac{\partial \varphi}{\partial x^{i}}\right\rangle,
$$

which imply that $\partial^{2} \varphi / \partial t \partial t=0$. Accordingly, there exist maps $\psi, \xi: M \rightarrow \boldsymbol{R}^{n+1}$ such that $\varphi(x, t)=t \psi(x)+\xi(x)$. We then find that $\xi$ is a constant map because

$$
\left(\left|\frac{\partial \psi}{\partial x^{i}}\right|^{2}-\kappa g_{i i}\right) t^{2}+2\left\langle\frac{\partial \psi}{\partial x^{i}}, \frac{\partial \xi}{\partial x^{i}}\right) t+\left|\frac{\partial \xi}{\partial x^{i}}\right|^{2}=0
$$

holds for any $t$ by the third equality of (5.7) and so $\left|\partial \xi / \partial x^{i}\right|=0$. Moreover, it follows from the first equality of (5.7) that $\psi(M) \subset S^{n}(1) \subset \boldsymbol{R}^{n+1}$.

In the second step, we claim that $c=0$ and $k=n+1$, so that the above diagram is commutative.

Since $\varphi_{*}(t T)=\sum_{j=1}^{n+1}\left(y^{j}-c^{j}\right) \partial / \partial y^{j}$ for $T=d / d t \in \Gamma(T \tilde{M})$, the third equality of (5.5) implies that

$$
\begin{aligned}
0 & =t^{2} \varphi_{*}\left\{\tilde{K}(T, T)+t^{-1} T\right\}=K_{k}\left(\varphi_{*}(t T), \varphi_{*}(t T)\right)+\varphi_{*}(t T) \\
& =\sum_{i, j=1}^{n+1}\left(y^{i}-c^{i}\right)\left(y^{j}-c^{j}\right) K_{k}\left(\frac{\partial}{\partial y^{i}}, \frac{\partial}{\partial y^{j}}\right)+\sum_{l=1}^{n+1}\left(y^{l}-c^{l}\right) \frac{\partial}{\partial y^{l}} \\
& =\sum_{l=n+1-k+1}^{n+1} \frac{\left(y^{l}-c^{l}\right)^{2}}{-y^{l}} \frac{\partial}{\partial y^{l}}+\sum_{l=1}^{n+1}\left(y^{l}-c^{l}\right) \frac{\partial}{\partial y^{l}} .
\end{aligned}
$$

Since the right-hand side can not vanish if $k<n+1$, we have $k=n+1$. Then the right-hand side is given by

$$
\sum_{l=1}^{n+1}\left\{\frac{\left(y^{l}-c^{l}\right)^{2}}{-y^{l}}+\left(y^{l}-c^{l}\right)\right\} \frac{\partial}{\partial y^{l}}=\sum_{l=1}^{n+1} c^{l} \frac{y^{l}-c^{l}}{y^{l}} \frac{\partial}{\partial y^{l}},
$$

which implies that $c=0$.

To sum up, we have $\varphi: \tilde{M} \rightarrow\left(\boldsymbol{R}^{+}\right)^{n+1}$ and $\iota: M \rightarrow S_{+}^{n}(\kappa)$ such that $\varphi^{*} D=\tilde{\nabla}$, $\varphi^{*} g_{n+1}=\widetilde{g}$ and $\varphi(x, t)=t \sqrt{\kappa} f \circ \iota(x)$, that is, $\varphi \circ \eta=f \circ \iota$. It is easy to show that $\iota^{*} \nabla^{S_{+}}=\nabla$ and $\iota^{*} g_{S_{+}}=g$.

\section{REFERENCES}

[ 1 ] S. Amari And H. Nagaoka, Methods of information geometry, Transl. Math. Monogr. 191, Amer. Math. Soc., Providence, RI; Oxford University Press, Oxford, 2000.

[2] H. Furuhata, Hypersurfaces in statistical manifolds, Differential Geom. Appl. 27 (2009), 420-429.

[ 3 ] T. Kurose, Geometry of statistical manifolds (in Japanese), Mathematics in the 21st century, 34-43, Nihonhyouron-sha, 2004. 
[4] H. Shima, Hessian manifolds of constant Hessian sectional curvature, J. Math. Soc. Japan 47 (1995), 735753.

[ 5 ] H. Shima, The geometry of Hessian structures, World Sci. Publ., Hackensack, NJ, 2007.

Department of Mathematics

HOKKAIDO UNIVERSITY

SAPPORO 060-0810

JAPAN

E-mail address: furuhata@math.sci.hokudai.ac.jp
Department of Mathematical Science

KWANSEI GAKUIN UNIVERSITY

SANDA 669-1337

JAPAN

E-mail address: crg31562@kwansei.ac.jp 